SUBJECT AREAS:

QUANTUM

INFORMATION

QUANTUM OPTICS

Received

29 October 2014

Accepted

19 February 2015

Published

20 March 2015

Correspondence and requests for materials should be addressed to R.-B.J. (ruibo@nict.go.

ip)

\section{Highly efficient entanglement swapping and teleportation at telecom wavelength}

\author{
Rui-Bo Jin ${ }^{1}$, Masahiro Takeoka', Utako Takagi ${ }^{1,2}$, Ryosuke Shimizu ${ }^{3} \&$ Masahide Sasaki'
}

\author{
${ }^{1}$ National Institute of Information and Communications Technology (NICT), 4-2-1 Nukui-Kitamachi, Koganei, Tokyo 184-8795, \\ Japan, ${ }^{2}$ Tokyo University of Science, 1-3 Kagurazaka, Shinjuku-ku, Tokyo 162-8601, Japan, ${ }^{3}$ University of \\ Electro-Communications, 1-5-1 Chofugaoka, Chofu, Tokyo 182-8585, Japan.
}

Entanglement swapping at telecom wavelengths is at the heart of quantum networking in optical fiber infrastructures. Although entanglement swapping has been demonstrated experimentally so far using various types of entangled photon sources both in near-infrared and telecom wavelength regions, the rate of swapping operation has been too low to be applied to practical quantum protocols, due to limited efficiency of entangled photon sources and photon detectors. Here we demonstrate drastic improvement of the efficiency at telecom wavelength by using two ultra-bright entangled photon sources and four highly efficient superconducting nanowire single photon detectors. We have attained a four-fold coincidence count rate of 108 counts per second, which is three orders higher than the previous experiments at telecom wavelengths. A raw (net) visibility in a Hong-Ou-Mandel interference between the two independent entangled sources was $73.3 \pm 1.0 \%(85.1 \pm 0.8 \%)$. We performed the teleportation and entanglement swapping, and obtained a fidelity of $76.3 \%$ in the swapping test. Our results on the coincidence count rates are comparable with the ones ever recorded in teleportation/swapping and multi-photon entanglement generation experiments at around $800 \mathrm{~nm}$ wavelengths. Our setup opens the way to practical implementation of device-independent quantum key distribution and its distance extension by the entanglement swapping as well as multi-photon entangled state generation in telecom band infrastructures with both space and fiber links.

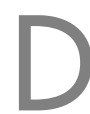

istribution of quantum entanglement through optical channels is the basis of implementing quantum information and communication protocols, which do not have any classical counterparts, such as deviceindependent quantum key distribution (DI-QKD) $)^{1,2}$, quantum secret sharing ${ }^{3,4}$, quantum repeater network ${ }^{5-9}$, and so on. The distance of direct transmission of entanglement is, however, severely limited, because the entanglement is easily destroyed by the channel loss and channel noises. Extending the distance and networking the entanglement requires entanglement swapping as the very elementary protocol. This is to convert two independent entangled photon pairs, say, photons $\mathrm{A}$ and $\mathrm{B}$, and $\mathrm{C}$ and $\mathrm{D}$, to a new entangled pair of photons between $\mathrm{A}$ and $\mathrm{D}$, those are not originally entangled, by performing a Bell measurement on photons B and C.

Practical methods for the swapping at present is to prepare entangled photons from spontaneous parametric down conversion (SPDC), to detect the two photons at the intermediate node by two single-photon detectors, and to herald the success event for the swapping. Thus the protocol is probabilistic. Its success rate is directly determined by the four-fold coincidence count (4-fold CC) rate.

The first entanglement swapping experiment was carried out in 1998, with SPDC process in BBO crystal ${ }^{10}$. Since then, many proof-of-principle experiments have been demonstrated at near infra-red wavelength range (around $800 \mathrm{~nm})^{9-11}$. Demonstrations at telecom wavelengths (around $1550 \mathrm{~nm}$ ) have also been demonstrated with SPDC in bulk crystals ${ }^{12-14}$, in waveguides ${ }^{15-17}$, or with spontaneous four-wave mixing (SFWM) in fibers ${ }^{18}$. Unfortunately, however, the efficiencies were very low, especially at telecom wavelengths so far. For example, the maximum 4 -fold CC rate has been 0.08 counts per second $(\mathrm{cps})^{14}$. This limits practical applications of entanglement swapping to quantum information and communication protocols.

In this work, we demonstrate highly efficient entanglement swapping by utilizing our high-quality entangled photon source ${ }^{19}$ and highly efficient superconducting nanowire single photon detectors (SNSPDs) ${ }^{20,21}$. In our experiment, 4-fold CC rate of $108 \mathrm{cps}$ was attained, which is three orders higher than the previous record ${ }^{14}$. A net visibility is $85.1 \pm 0.8 \%$ in Hong-Ou-Mandel interference between two independent entangled sources. We also demonstrate high quality teleportation experiment, with 2 -fold CC of $150 \mathrm{kcps}$, which is comparable to those obtained in highly efficient schemes in the near-infrared wavelength ${ }^{22-25}$, and with the entanglement visibility of $98 \%$, which is the highest among ${ }^{22-25}$. 


\section{Experiment and Results}

The experimental setup is shown in Fig. 1. The entangled photon source is based on a SPDC from a group-velocity-matched periodically poled $\mathrm{KTiOPO}_{4}$ (GVM-PPKTP) crystal in a Sagna-loop configuration $^{19}$. This entangled photon source has a spectral purity as high as $0.82^{26}$, which can widen applications with multi-source of entangled photons. The SNSPD has a maximum system detection efficiency (SDE) of $79 \%$ with a dark count rate (DCR) of $2 \mathrm{kcps}$. For more details of this Saganc-GVM-PPKTP entangled source, refer to Refs. $19,27,28$. By carefully improving the coupling efficiency, we have improved the coincidence counts from $20 \mathrm{kcps}$ (in Ref. 19) to $40 \mathrm{kcps}$, at $10 \mathrm{~mW}$ pump power. The overall efficiency, which is the product of all efficiencies between the source and detectors, was improved from 0.10 (in Ref. 19) to 0.20 . Further, the minimal interference visibility in polarization correlation measurement was improved from $\sim 96 \%$ (in Ref. 19 ) to $\sim 98 \%$ at $10 \mathrm{~mW}$ pump power, by finely optimizing the alignment in the Sagnac-loop.

The entangled source. First, we perform the photon polarization correlation measurement with the setup shown in Fig. 1(a). Both

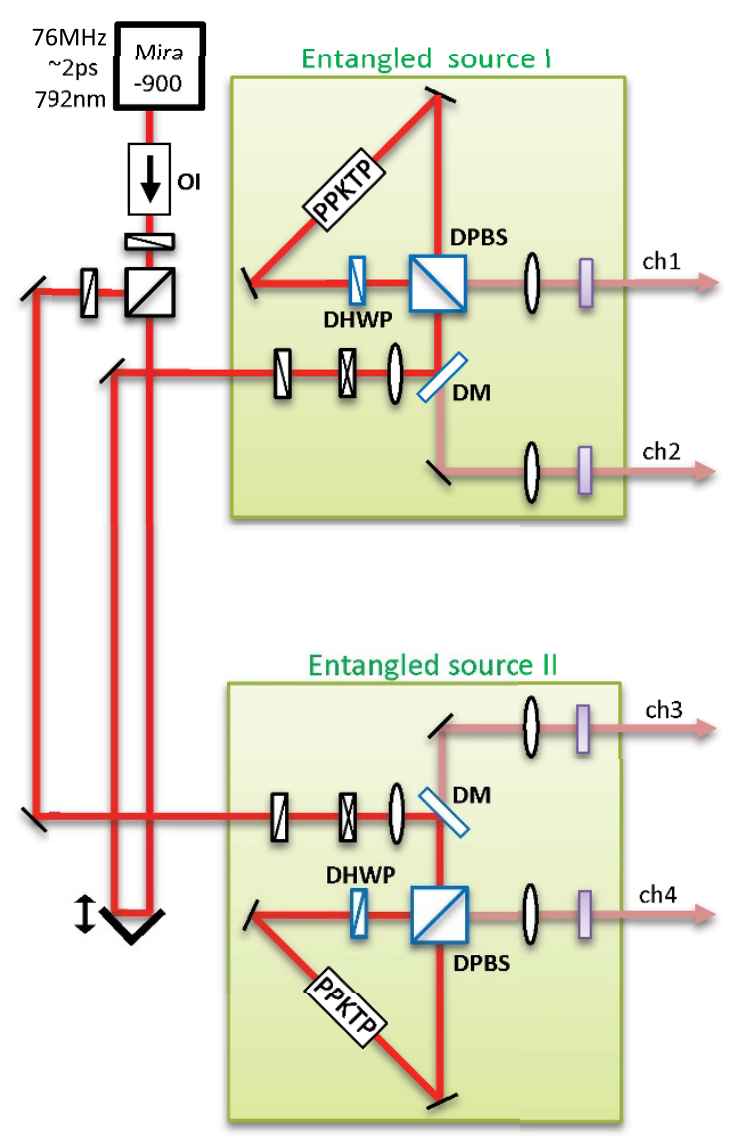

(a)

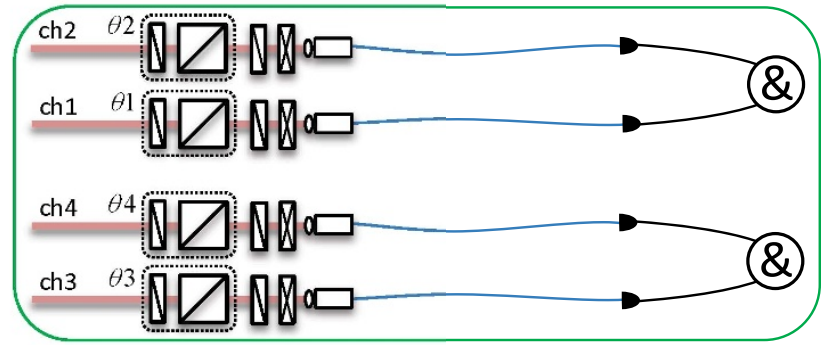

(b)

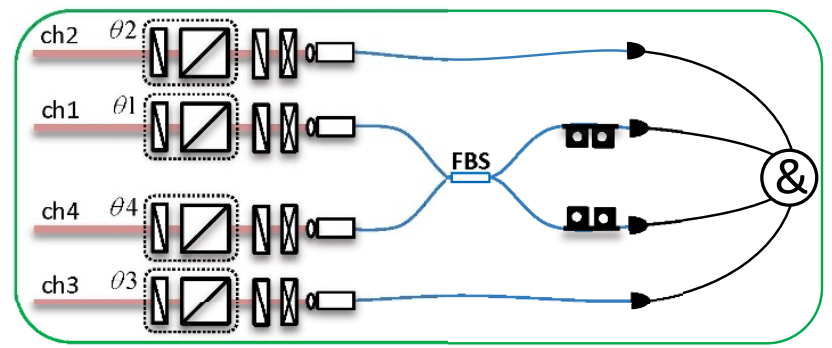

(c)

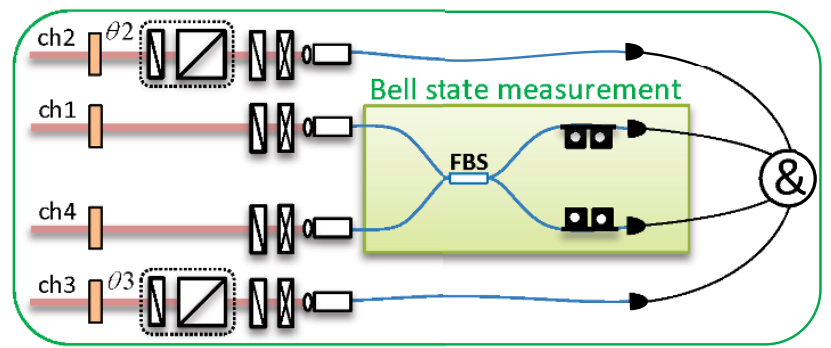

(d)

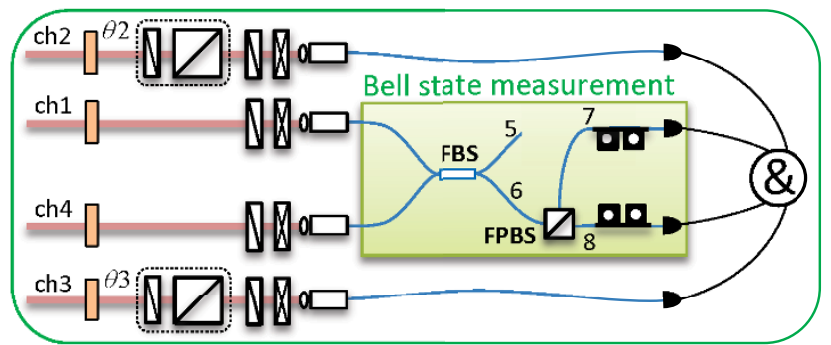

Figure 1 The experimental setup. Picosecond laser pulses (76 MHz, $792 \mathrm{~nm}$, temporal duration $\sim 2 \mathrm{ps}$ ) from a mode-locked Titanium sapphire laser (Mira900, Coherent Inc.) were divided into two paths, and pumped two entangled photon sources which were in Sagnac-loop configurations and focused by two $f=200 \mathrm{~mm}$ lenses (beam waist $\sim 45 \mu \mathrm{m}$ ). Each Sagnac-loop consisted of a dichroic mirror (DM), a dual-wavelength polarization beam splitter (DPBS), a dual-wavelength half-wave plate (DHWP), and a 30-mm-long PPKTP crystal with a polling period of $46.1 \mu \mathrm{m}$ for a type-II collinear groupvelocity-matched SPDC. The downconverted photons passed through four sets of longpass filters (LPF), four polarizers $\left(\theta_{1}\right.$ to $\left.\theta_{4}\right)$, four sets of half-wave plate (HWP) and quarter-wave plate (QWP), and then coupled into four channels (ch1 to ch4) of single-mode fibers. Two sets of fiber polarization controllers (FPC) were used to optimize the polarization of the photons before detected by SNSPDs, which were connected to a coincidence counter (\&). $\theta_{1}$ and $\theta_{4}$ were removed in the tests of teleportation and entanglement swapping, so as to couple both the horizontal $(\mathrm{H})$ and vertical $(\mathrm{V})$ polarized photons into the fiber beam splitter (FBS, Thorlabs, PBC1550SM-FC). (a) is for entangled source test. (b) is for Hong-Ou-Mandel interference test. (c) is for teleportation test. (d) is for entanglement swapping test. In the teleportation test in (c), Bell state measurement (BSM) was realized by a FBS. To overcome the polarization dependance of the SNSPDs in entanglement swapping test in (d), the BSM was realized by using the combination of a FBS and a fiber based PBS (FPBS, PBC1550SM-FC, Thorlabs), which was constituted of a calcite prism and input/output fibers. Four coarse bandpass filters (CBPF) were inserted in four channels (ch1-ch4) for the tests of teleportation and entanglement swapping. Note that the order of the channels was not the same for the main panel (ch1, ch2, ch3, ch4, from top to bottom) and panels (a)-(d) (ch2, ch1, ch4, ch3). Due to the technical reason, ch1 and ch4 were guided into the FBS. 
the source I and source II are prepared in $\left|\psi^{-}\right\rangle=\frac{1}{\sqrt{2}}(|H V\rangle-|V H\rangle)$ state. With pump powers of $80 \mathrm{~mW}$ for source I and $85 \mathrm{~mW}$ for source II, we achieve coincidence counts of around $150 \mathrm{kcps}$, as shown in Fig. 2, corresponding to $300 \mathrm{kcps}$ without the polarizers. The corresponding mean photon-pair numbers per pulse are around 0.1 for both sources. The raw visibilites are around $87 \%$, while the background subtracted visibilities (i.e., net visibilities) are around $98 \%$ for each polarizers set for source II. The degradation of this visibility at high pump power is mainly caused by the multi-pair emission. The result agrees with the theoretical model in Ref. 28 which includes multi-photon emissions and system imperfections (see Methods). These high-brightness entangled photon sources guarantee the high count rate in the following teleportation and entanglement swapping experiments.

Hong-Ou-Mandel interference. Next, we measure a four-fold Hong-Ou-Mandel (HOM) interference ${ }^{29}$ between ch1 and ch4 (heralded by ch2 and ch3) with 4 polarizers inserted in each channel in Fig. 1(b). The polarization angles for $\theta_{1}-\theta_{4}$ are set at $0 \%$ $90^{\circ} / 90^{\circ} / 0^{\circ}$, respectively. Firstly, we test the HOM interference with no bandpass filters inserted, whose result is shown in Fig. 3(a). The 4-fold CC is 169 cps (5080 counts in $30 \mathrm{~s}$ ) and the raw visibility is $67.1 \pm 0.9 \%$. The background counts is mainly caused by the multipair emission in SPDC. We subtract the background counts using the same method as shown in Ref. 30 . We block only ch1 and measure 4fold CCs, then block only ch4, and measure 4 -fold CCs. The sum of these two coincidence counts constitutes the background count. After background subtraction, the net visibility is $78.4 \pm 0.8 \%$, which is consistent with our previous results in Ref. 30 .

To further increase the visibility, we should improve the spectral purity of photons. As reported in Ref. 26, the downconverted photons from our PPKTP crystal have an intrinsic spectral purity of 0.82 , and this value can be improved to unity by inserting coarse bandpass filters (C-BPFs) to cut the side lobes in the joint spectral amplitude. In this experiment, we prepared four C-BPFs which have near-Gaussian shape with FWHM (full width at half maximum) of $2.1 \mathrm{~nm}$ and peak transmission efficiency of $93 \%$ at the central wavelength of $1584 \mathrm{~nm}$. The overall transmittance efficiency of the CBPFs is around $77 \%$, tested with our downconverted photons, which have spectra of Gaussian shape with FWHM of $1.2 \mathrm{~nm}$ and center wavelength of $1584 \mathrm{~m}$. We insert two C-BPFs in ch1 and ch4 and repeat the HOM interference, whose result is shown in Fig. 3(b). The 4-fold CC rate is $108 \mathrm{cps}$ ( 3249 counts in $30 \mathrm{~s}$ ) and the raw visibility is $73.3 \pm 1.0 \%$. After subtracting the background multi-photon emission, the net visibility is $85.1 \pm 0.8 \%$. The raw (net) visibility was improved by $6.2 \%(6.7 \%)$ after the insertion of the two C-BPFs, due to the increase of the spectral purity. However, this visibility improvement is lower than our expectation (net visibility $\approx 100 \%$ ),

\section{(a) Soucre I}

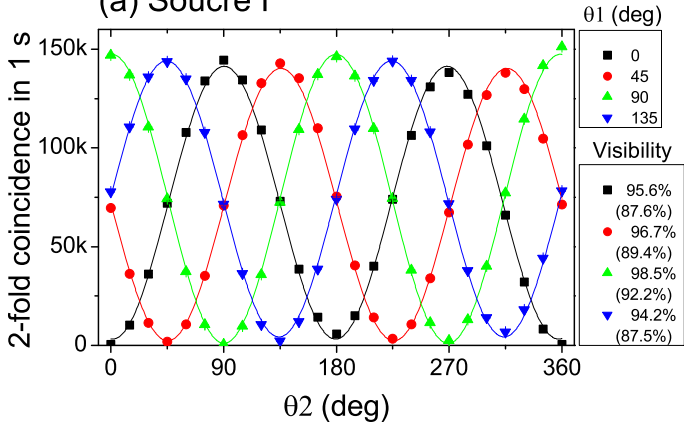

which may be caused by the following reasons: the photons generated from two different nonlinear crystals may have different spectral properties; the transmission profiles of these C-BPFs can not be perfectly the same, which may also lead to the difference of the transmitted photons; a small portion of the side-lodes may be not filtered because the transmission shape of the C-BPFs is not in a perfect Gaussian-shape.

We also investigate the HOM interference with four C-BPFs inserted in each channel, whose result is shown in Fig. 3(c). The 4 -fold CC rate is $78 \mathrm{cps}$ (2329 in $30 \mathrm{~s}$ ) and the raw (net) visibility is $75.6 \pm 1.1 \%(87.2 \pm 0.8 \%)$. The raw (net) visibility was improved by $2.3 \%(2.1 \%)$ after the insertion of these two more C-BPFs. In the following test of quantum teleportation and entanglement swapping, all these four C-BPFs are inserted.

Quantum teleportation. After the test of Hong-Ou-Mandel interference, we remove Polarizer $1\left(\theta_{1}\right)$ and Polarizer $4\left(\theta_{4}\right)$, then the setup in Fig. 1(c) is ready for the test of quantum teleportation. The principle of teleportation in our experiment is as follow. Assume photons in ch1 are in an initial state of $|i\rangle_{1}=(\alpha|H\rangle+\beta|V\rangle)_{1}$, and photons in ch3 and ch4 are in an entangled state of $\left|\psi^{-}\right\rangle_{34}=\frac{1}{\sqrt{2}}(|H V\rangle-|V H\rangle)_{34}$. A partial Bell state measurement on photons in ch1 and ch4 will teleport the state $|i\rangle_{1}$ to the photons in ch3, i.e., the final state of the photons in ch3 will be $|f\rangle_{3}=(\alpha|H\rangle+\beta|V\rangle)_{3}$. This process can be expressed as ${ }^{31}$ :

$$
\begin{aligned}
& \left|\psi^{-}\right\rangle_{34} \otimes|i\rangle_{1}=\frac{1}{\sqrt{2}}(|H V\rangle-|V H\rangle)_{34}(\alpha|H\rangle+\beta|V\rangle)_{1} \\
& =\frac{1}{2}\left[\begin{array}{c}
\left|\psi^{+}\right\rangle_{41}(-\alpha|H\rangle+\beta|V\rangle)_{3}+\left|\psi^{-}\right\rangle_{41}(\alpha|H\rangle+\beta|V\rangle)_{3} \\
+\left|\phi^{+}\right\rangle_{41}(\alpha|V\rangle-\beta|H\rangle)_{3}+\left|\phi^{-}\right\rangle_{41}(\alpha|V\rangle+\beta|H\rangle)_{3}
\end{array}\right]
\end{aligned}
$$

where $\left|\psi^{ \pm}\right\rangle=\frac{1}{\sqrt{2}}(|H V\rangle \pm|V H\rangle)$ and $\left|\phi^{ \pm}\right\rangle=\frac{1}{\sqrt{2}}(|H H\rangle \pm|V V\rangle)$ are the four Bell states. We only focus the second term:

$$
\left|\psi^{-}\right\rangle_{34} \otimes|i\rangle_{1} \rightarrow\left|\psi^{-}\right\rangle_{41} \otimes|f\rangle_{3}
$$

In this process, the partial Bell state measurement is realized by coincidence counting after a beam splitter (i.e., FBS in Fig. 1(c)), due to the fact that only one input state, $\left|\psi^{-}\right\rangle$, out of the four Bell states has coincidence counts after the beam splitter. The state of the photons in ch1 is heralded by the photon states in ch2 with the correlation of $\left|\psi^{-}\right\rangle_{12}=\frac{1}{\sqrt{2}}(|H V\rangle-|V H\rangle)_{12}=\frac{1}{\sqrt{2}}(|A D\rangle-|D A\rangle)_{12}$, where,
$|D\rangle=\frac{1}{\sqrt{2}}(|H\rangle+|V\rangle) \quad$ (corresponding to $\theta=45^{\circ}$ ) and
$|A\rangle=\frac{1}{\sqrt{2}}(|H\rangle-|V\rangle)$ (corresponding to $\theta=135^{\circ}$ ). (b) Source II

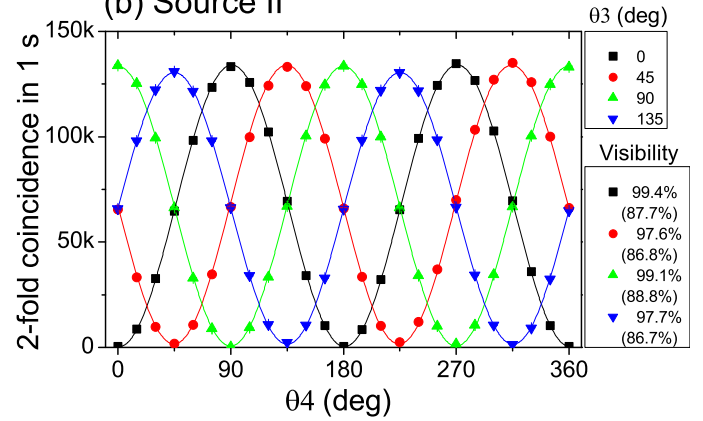

Figure $2 \mid$ Two-fold coincidence counts in one second as a function of the two polarizers, with a pump power of $80 \mathrm{~mW}$ for entangled source I (a) and $85 \mathrm{~mW}$ for entangled source II (b). Both the source I and source II were prepared in $\left|\psi^{-}\right\rangle=\frac{1}{\sqrt{2}}(|H V\rangle-|V H\rangle)$ state. The background subtracted visibilities (raw visibilities) are shown on the right side. The error bars were added by assuming Poissonian statistics of these coincidence counts. 

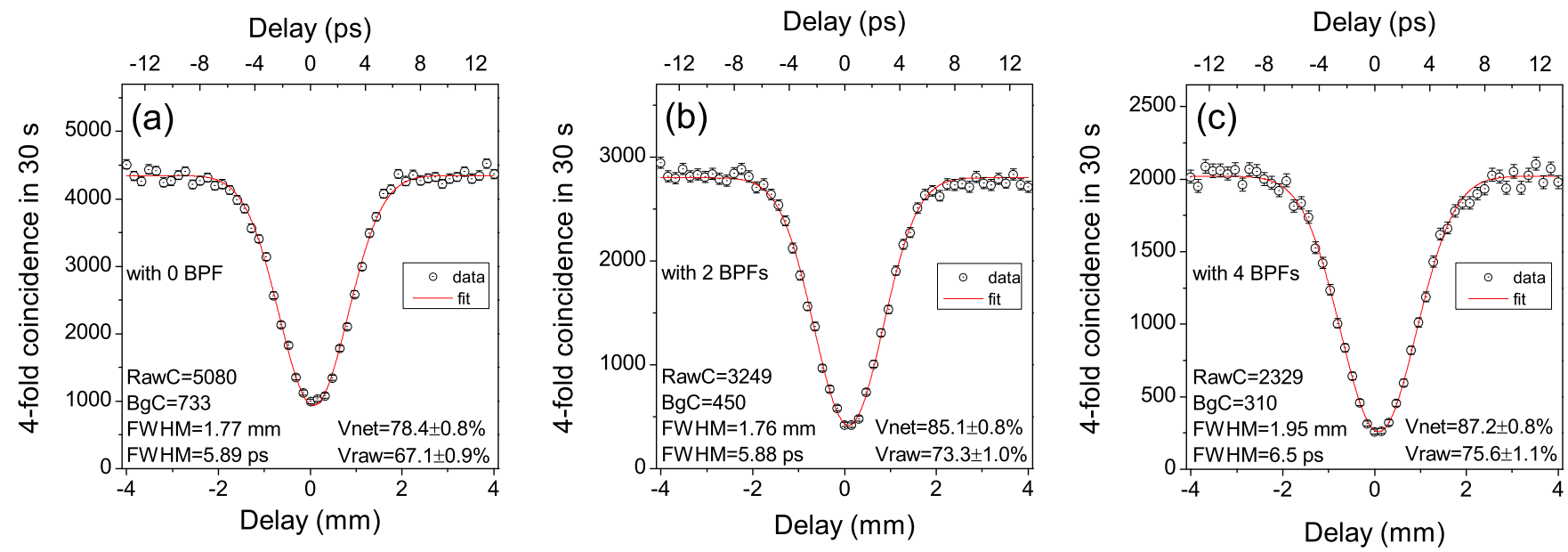

Figure 3 Hong-Ou-Mandel interference. (a) with no C-BPF, (b) with two C-BPFs, (c) with four C-BPFs. The error bars were calculated by assuming Poissonian statistics of these coincidence counts.

First, we demonstrate a teleportation in H/V bases, as shown in Fig. 4(a) and (b). The initial state of the photons in ch1 is in $\mathrm{H}$ polarization, i.e., $|i\rangle_{1}=|H\rangle_{1}$, which is heralded by their daughter photons in ch2 with $\mathrm{V}$ polarization $\left(\theta_{2}=90^{\circ}\right)$. Then, the partial Bell state measurement on chl and ch4 projects the correlated photons in ch3 with $\mathrm{H}$ polarization, i.e., $|f\rangle_{3}=|H\rangle_{3}$. With this condition, if the angle of Polarizer 3 is $\theta_{3}=0^{\circ}$, the 4 -fold CC exists, therefore, no HOM dip appears at the zero delay point, as shown in Fig. 4(b). Otherwise, if $\theta_{3}=90^{\circ}$, all the H-polarized photons are blocked, then ideally, there is no 4 -fold CC at the zero delay point, so a HOM dip occurs, as shown in Fig. 4(a). Similarly, we also showed the teleportation results with other bases, as shown in Fig. 4(c) and (d) for ch1 at $|D\rangle$ bases (with $\theta_{2}=45^{\circ}$ ). More results are summarized in Tab. 1. The visibilities in $\mathrm{H} / \mathrm{H}, \mathrm{V} / \mathrm{V}, \mathrm{A} / \mathrm{A}, \mathrm{D} / \mathrm{D}$ bases range from $75.8 \%$ to $84.9 \%$, all well beyond the classical limit of $50 \%$. These results demonstrate the potential of our setup for highly efficient quantum teleportation. To fully verify the nonclassicality of quantum teleportation, one has to perform the experiment with two more states $((|H\rangle \pm i|V\rangle) / \sqrt{2})$.

It should be noted that from the coincidence counts in Fig. 4(a-d), we can see the SNSPDs are strongly polarization-dependent. Because of its special construction structure, the SNSPD has a maximal efficiency on certain polarization direction and its orthogonal direction has the minimal efficiency. According to our experimental tests, the maximal efficiency is typically two times of the minimal efficiency. To avoid the polarization-dependency of the SNSPDs for the entanglement swapping test, we change the FBS in Fig. 1(c) to the combination of a FBS and a FPBS in Fig. 1(d).
Entanglement swapping. The principle of entanglement swapping can be understood from the following equation ${ }^{32}$ :

$\left|\psi^{-}\right\rangle_{12} \otimes\left|\psi^{-}\right\rangle_{34}=\frac{1}{2}\left(\begin{array}{l}\left|\psi^{+}\right\rangle_{14} \otimes\left|\psi^{+}\right\rangle_{23}-\left|\psi^{-}\right\rangle_{14} \otimes\left|\psi^{-}\right\rangle_{23} \\ -\left|\phi^{+}\right\rangle_{14} \otimes\left|\phi^{+}\right\rangle_{23}+\left|\phi^{-}\right\rangle_{14} \otimes\left|\phi^{-}\right\rangle_{23}\end{array}\right)$

The detection of an entangled state in ch1 and ch4 heralds the existence of entanglement in ch2 and ch3, which originally have no correlation. The partial Bell state measurement in Fig. 1(d) is realized by the combination of FBS and FPBS. Only one input state $\left|\psi^{+}\right\rangle$out of the four Bell states has coincidence counts at port 7 and port 8 in Fig. 1(d), due to the transformation of a BS: $\left|\psi^{+}\right\rangle_{14}=\frac{1}{\sqrt{2}}\left(\left|H_{1} V_{4}\right\rangle+\left|V_{1} H_{4}\right\rangle\right) \rightarrow \frac{1}{\sqrt{2}}\left(\left|H_{5} V_{5}\right\rangle-\left|V_{6} H_{6}\right\rangle\right)$.

To realize such a scheme in experiment, we need to firstly calibrate the photon polarizations in the FBS and FPBS. We reinsert Polarizer 1 and Polarizer 4, and rotate the angles of HWPs and QWPs in ch1 and ch4, so as to achieve the following condition: the $\mathrm{H}$ polarized photons in ch 1 travel to outport 8 , while the $\mathrm{H}$ polarized photons in ch4 travel to outport 7. Under this condition, the $\mathrm{H}(\mathrm{V})$ polarized photons in ch4 are converted to $\mathrm{V}(\mathrm{H})$ polarized, while the polarization of photons in ch1 is not changed and hence can function as a reference. Therefore, an input state of $\left|\phi^{ \pm}\right\rangle_{14}$ is transformed to the state of $\left|\psi^{ \pm}\right\rangle_{14}$, and the $\left|\psi^{ \pm}\right\rangle_{14}$ state is transform to $\left|\phi^{ \pm}\right\rangle_{14}$ state, respectively. As a result, the state in Eq. (3) is transformed to Eq. (4):

$$
\left|\psi^{-}\right\rangle_{12} \otimes\left|\psi^{-}\right\rangle_{34} \rightarrow \frac{1}{2}\left(\begin{array}{l}
\left|\phi^{+}\right\rangle_{14} \otimes\left|\psi^{+}\right\rangle_{23}-\left|\phi^{-}\right\rangle_{14} \otimes\left|\psi^{-}\right\rangle_{23} \\
-\left|\psi^{+}\right\rangle_{14} \otimes\left|\phi^{+}\right\rangle_{23}+\left|\psi^{-}\right\rangle_{14} \otimes\left|\phi^{-}\right\rangle_{23}
\end{array}\right)(4)
$$
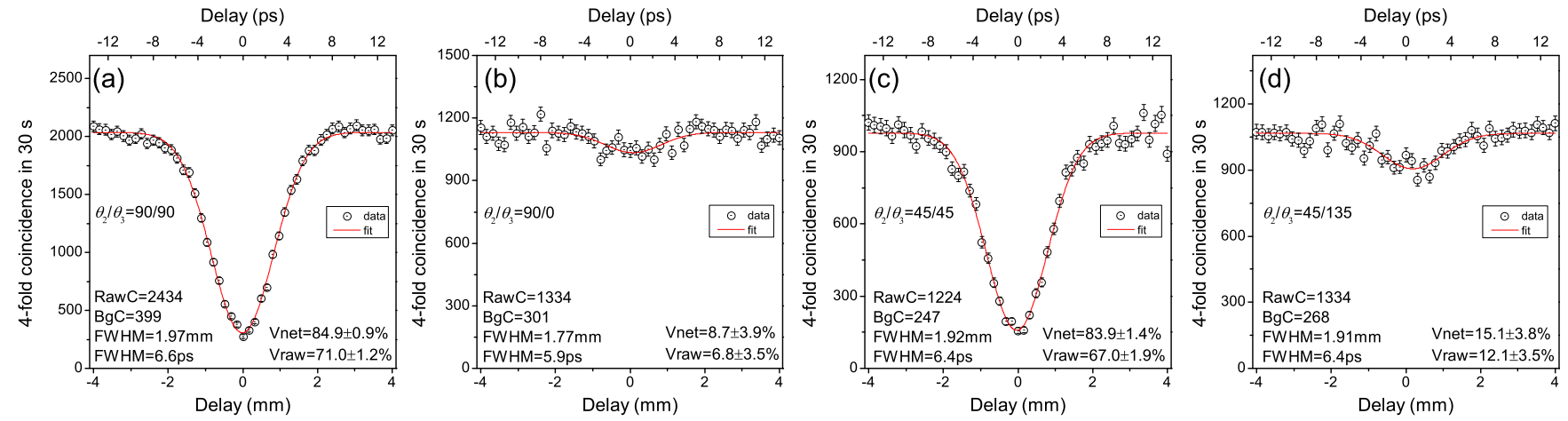

Figure $4 \mid$ Teleportation with Polarizers $1 / 4$ removed, and Polarizers $2 / 3$ at $90^{\circ} / 90^{\circ}, 90^{\circ} / 0^{\circ}, 45^{\circ} / 45^{\circ}$ and $45^{\circ} / 135^{\circ}$ for (a-d), respectively. The uncertainties of these visibilities were derived using Poissonian errors on the coincidence counts. 
Table 1 | Quantum teleportation at different angles of Polarizer $2\left(\theta_{2}\right)$ and Polarizer $3\left(\theta_{3}\right)$

\begin{tabular}{lcrr}
$\theta_{2} / \theta_{3}$ & $\mathrm{~V}_{\text {net }}\left(\mathrm{V}_{\text {raw }}\right)$ & $\theta_{2} / \theta_{3}$ & $\mathrm{~V}_{\text {net }}\left(\mathrm{V}_{\text {raw }}\right)$ \\
\hline $0^{\circ} / 0^{\circ}$ & $75.8 \pm 2.6 \%(55.9 \pm 3.2 \%)$ & $45^{\circ} / 45^{\circ}$ & $83.9 \pm 1.4 \%(67.0 \pm 1.9 \%)$ \\
$0^{\circ} / 90^{\circ}$ & $14.2 \pm 4.1 \%(11.4 \pm 3.8 \%)$ & $45^{\circ} / 135^{\circ}$ & $15.1 \pm 3.8 \%(12.1 \pm 3.5 \%)$ \\
$90^{\circ} / 0^{\circ}$ & $8.7 \pm 3.9 \%(6.8 \pm 3.5 \%)$ & $135^{\circ} / 45^{\circ}$ & $16.9 \pm 3.5 \%(13.2 \pm 3.2 \%)$ \\
$90^{\circ} / 90^{\circ}$ & $84.9 \pm 0.9 \%(71.0 \pm 1.2 \%)$ & $135^{\circ} / 135^{\circ}$ & $81.9 \pm 1.3 \%(65.9 \pm 1.7 \%)$ \\
\hline
\end{tabular}

Let us focus on the third term. The $\left|\psi^{+}\right\rangle_{14}$ state will be detected by the Bell state analyzer, and thus projects the state in ch2 and ch3 to $\left|\phi^{+}\right\rangle_{23}$ state.

After the calibration, Polarizer 1 and Polarizer 4 are removed. We fix the optical path delay at the zero delay position, then we rotate $\theta_{2} /$ $\theta_{3}$ and record the 4 -fold CC, whose result is shown in Fig. 5 (a). Figure 5 (a) shows an experimental interference pattern of $\left|\phi^{+}\right\rangle_{23}$ state, which is consistent with our theoretical expectation in Eq. (4). The net (raw) visibilities at $\theta_{2}=0^{\circ} / 45^{\circ} / 90^{\circ} / 135^{\circ}$ are $90.6 \%$ (78.0\%)/65.7\% (56.1\%)/87.4\% (74.6\%)/63.6\% (54.4\%), respectively. The background counts are subtracted using the same method as described in the four-fold HOM interference.

To decrease the effect of multi-photon emission, we decrease the pump power in Fig. 5(b) to half of the power in Fig. 5(a), i.e., the pump power is $40 \mathrm{~mW}$ for source I and $42.5 \mathrm{~mW}$ for source II in Fig. 1 (d). We find the raw visibilities at $0^{\circ} / 45^{\circ} / 90^{\circ} / 135^{\circ}$ in Fig. $5(\mathrm{a})$ were improved by $9.5 \%, 9.8 \%, 10.1 \%$ and $8.9 \%$ from Fig. 5(a).

The difference of the maximum counts at four different polarizer angles in Fig. 5 might be affected by the relative phase $\delta$ in the entangled state $\left|\phi_{23}^{+}\right\rangle=\frac{1}{\sqrt{2}}\left(\left|H_{2} H_{3}\right\rangle+e^{i \delta}\left|V_{2} V_{3}\right\rangle\right)$. Such a relative phase might be caused by the imperfections of our setup, for example, the spectral mode matching between $\mathrm{H}$-polarized photons and V-polarized photons; the slight difference of the quantum efficiency of the four SNSPDs; the splitting ratio of the BS was not exactly $50 / 50$.

All the visibilities in Fig. 5(a,b) are higher than 1/3, verifying the photons are entangled, according to the Peres criteria ${ }^{33}$. The minimal visibility (V) in Fig. 5(b) is $68.4 \%$, corresponding to a minimal fidelity (F) of $76.3 \%$, by considering the relation $4 F=3 V+1^{13}$.

\section{Discussion}

Comparison of brightness. We achieved a four-fold coincidence count rate of around $100 \mathrm{cps}$, and a raw (net) visibility of $73.3 \pm$ $1.0 \%(85.1 \pm 0.8 \%)$ in the experiment. This count rate is 3 orders higher than the previous experiments at telecom wavelength ${ }^{12-18,34,35}$. We compare the brightness of our result with the previous ones at telecom wavelengths in Tab. 2. There are mainly three reasons for this big technical jump in our high four-fold coincidence count rates. The first one attributes to the intrinsic high spectral purity of our

(a) pump $=80 \mathrm{~mW}$

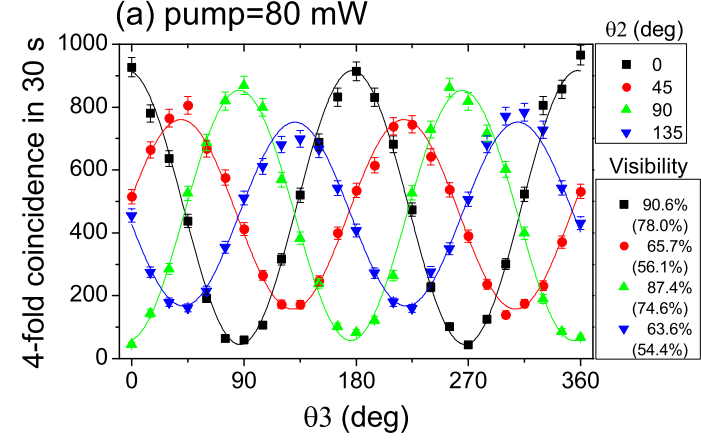

source. Thanks to the group-velocity matching condition, the intrinsics purity is as high as 0.82 , which is much higher than the conventional purity of PPLN crystal. Therefore, there is no need for narrow bandpass filters, which are widely used in conventional scheme and decrease the brightness of the source severely. The second reason comes from the optimization of the alignment, especially, the improvement of the coupling efficiency to the single mode fibers for both the clockwise pump and counter clockwise pump in the Sagnac-loop. The last and also the most important reason is the high efficiency of our SNSPDs, which showed 30 times higher count rates than the traditional InGaAs avalanche photodiode $(\mathrm{APD})^{36}$.

Application for field test in free space and fibers. We noticed that our count rates and visibilities are comparable to the previous teleportation and entanglement swapping experiments over $100 \mathrm{~km}$ free space channel at $\sim 800 \mathrm{~nm}$ wavelengths ${ }^{22,23,37}$, as compared in Tab. 3. Therefore, our scheme is directly applicable to the longdistance field test of teleportation and entanglement swapping at telecom wavelengths, which is the heart of a global quantum internet $^{22,38,39}$. Although conventional systems using BBO crystals and Si-APDs are applicable to free space communications, the BBO sources operating at $800 \mathrm{~nm}$ band are never applied to fiber communications. With high count rates and visibilities, our source can demonstrate the practical quantum communications using fiber infrastructures. It is also possible to combine both the free space links and fiber links using our scheme. For example, after free-space transmission for a long distance, the photons can be directly collected into fibers for further fiber transmission.

Application for 6, 8-photon entangled state. The brightness of our photon source is also comparable to the previous eight-photon entangled state generation experiments at $\sim 800 \mathrm{~nm}$ wavelength $\mathrm{s}^{24,25}$, as in Tab. 3. So our source can also be expanded to generate the 6, 8photon entangled state at telecom wavelengths. All the photon sources in Refs. 22-25, 37 were pumped by the second harmonic of the fundamental Ti:Sapphire lasers operating at $\sim 76 \mathrm{MHz}$ repetition rate. In contrast, our photon source doesn't require a secondharmonic process, which has a limited efficiency. Therefore, our scheme is possible to achieve higher count rate, especially when pumped by high-repetition rate lasers ${ }^{40}$.

(b) pump $=40 \mathrm{~mW}$

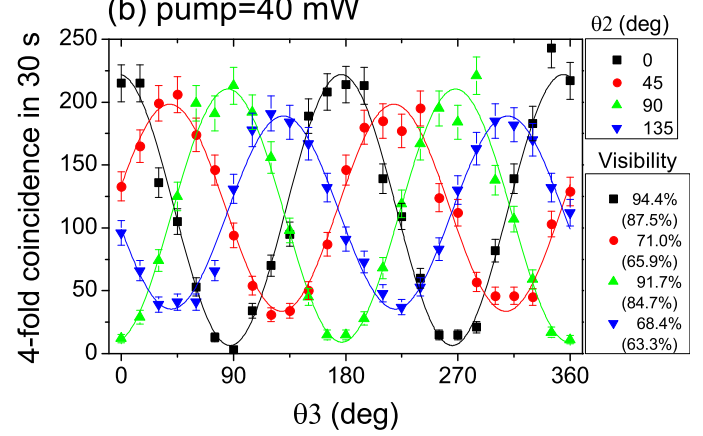

Figure 5 The entanglement swapping result. (a) with 80 (85) $\mathrm{mW}$ pump power for entangled source I (II). (b) the power was reduced to 40 (42.5) $\mathrm{mW}$ for entangled source I (II). The background subtracted visibilities (raw visibilities) are shown on the right side. The error bars were added by assuming Poissonian statistics of these coincidence counts. 
Table 2 | Comparison of wavelength, photon source, four-fold coincidence count rate (4-fold CC), raw visibility (V-raw) and net visibility (Vnet) with the previous teleportation or entanglement swapping experiments at telecom wavelengths. $V_{\text {telep., }} V_{\text {swap. }}$ and $V_{\text {HOM }}$ are the visibilities in teleportation, entanglement swapping and Hong-Ou-Mandel interference tests, respectively

\begin{tabular}{|c|c|c|c|c|c|c|c|c|}
\hline Reference & Group & wavelength & source & 4-fold CC & V-raw & V-net & qubit type & application \\
\hline Marcikic2003 $3^{12}$ & Geneva & $1310 / 1550 \mathrm{~nm}$ & LBO & $0.05 \mathrm{cps}$ & $\mathrm{V}_{\text {telep. }}=70 \%$ & NA & time-bin & teleportation \\
\hline Riedmatten $2005^{13}$ & Geneva & $1310 / 1550 \mathrm{~nm}$ & $\mathrm{LBO}$ & $0.0037 \mathrm{cps}$ & $V_{\text {swap. }}=80 \%$ & NA & time-bin & swapping \\
\hline Halder2007 & Geneva & $1560 \mathrm{~nm}$ & PPLN-WG & $0.0003 \mathrm{cps}$ & $\mathrm{V}_{\text {HOM }}=77 \%$ & NA & time-bin & swapping \\
\hline Xue201216,17 & Tsukuba & $1538 / 1562 \mathrm{~nm}$ & PPLN-WG & $0.016 \mathrm{cps}$ & $\mathrm{V}_{\text {HOM }}=75 \%$ & NA & polarization & swapping \\
\hline Wu2013 14 & Tokyo & $1550 \mathrm{~nm}$ & PPLN & $0.08 \mathrm{cps}$ & $\mathrm{V}_{H O M}=\mathrm{NA}$ & $92 \%$ & polarization & swapping \\
\hline This work & Tokyo & $1584 \mathrm{~nm}$ & PPKTP & 108 cps & $V_{\text {HOM }}=73 \%$ & $85 \%$ & polarization & swap./telep. \\
\hline
\end{tabular}

Table 3 | Comparison with the previous entangled source at NIR wavelengths. $V_{\text {ent. }}$ is visibility of the entangled (ent.) state in a correlation measurement. $C_{2(4)}$ is the 2(4)-fold coincidence count rate

\begin{tabular}{|c|c|c|c|c|c|}
\hline Reference & Group & wavelength & source & 2(4)-fold CC and visibility & application \\
\hline $\begin{array}{l}\text { Herbst2014 } \\
\text { Yin2012 } \\
\text { Yao2012 } \\
\text { Huang201 } 1^{25} \\
\text { This work }\end{array}$ & $\begin{array}{l}\text { Vienna } \\
\text { Hefei } \\
\text { Shanghai } \\
\text { Hefei } \\
\text { Tokyo }\end{array}$ & $\begin{array}{l}808 \mathrm{~nm} \\
808 \mathrm{~nm} \\
780 \mathrm{~nm} \\
780 \mathrm{~nm} \\
\mathbf{1 5 8 4} \mathbf{~ n m}\end{array}$ & $\begin{array}{l}\mathrm{BBO} \\
\mathrm{BBO} \\
\mathrm{BBO} \\
\mathrm{BBO} \\
\text { PPKTP }\end{array}$ & $\begin{array}{l}\mathrm{C}_{2}=130 \mathrm{kcps}, \mathrm{C}_{4}=100 \mathrm{cps}, \mathrm{V}_{\text {swap. }}=60 \% \\
\mathrm{C}_{2}=440 \mathrm{kcps}, \mathrm{V}_{\text {ent. }}=91 \%, \mathrm{~V}_{\text {HOM }}=60 \% * \\
\mathrm{C}_{2}=310 \mathrm{kcps}, \mathrm{V}_{\text {ent. }}=94 \%, V_{\text {HOM }}=76 \% \\
\mathrm{C}_{2}=220 \mathrm{kcps}, V_{\text {ent. }}=97 \%, V_{\text {HOM }}=82 \% \\
\mathbf{C}_{\mathbf{2}}=\mathbf{1 5 0} \text { kcps, } \mathbf{V}_{\text {ent. }}=\mathbf{9 8 \%}, \mathbf{V}_{\text {HOM }}=\mathbf{7 8 \%}\end{array}$ & $\begin{array}{l}\text { swapping } 143 \mathrm{~km} \\
\text { teleportation } 100 \mathrm{~km} \\
\text { 8-photon ent. state } \\
\text { 8-photon ent. state } \\
\text { swap./telep. }\end{array}$ \\
\hline
\end{tabular}

Application for quantum key distribution (QKD). Our setup opens the way to practical implementation of the qubit-amplifier based device-independent-QKD scheme, which was proposed by Gisin and colleagues in $2010^{41}$. Furthermore, our result shows a potential toward the realization of the entanglement swapping based QKD (ES-QKD) protocols ${ }^{42,43}$. In our experiment, we achieved the 4-fold CC rate of over $100 \mathrm{cps}$ which could be still meaningful value even in lossy channels. For example, adding a total loss of $10 \mathrm{~dB}$ (50 km distance in standard fiber) in our system, the 4 -fold CC rate remains 10 cps. This is still comparable to the recent field demonstration of the entanglement based QKD in Ref. 44 which suggests that our count rate is enough to realize the first demonstration of the ES-QKD.

Application for quantum repeater. In the scenario of quantum repeaters, a point to point quantum communication between remote locations is limited to about $300-500 \mathrm{~km}$ due to the losses in fibers, but this problem can be solved by decompose the long distance into serval shorter elementary links. In each link, the entanglement is shared and stored in quantum memories with long coherence time. Finally, the entangled state is retrieved from the quantum memories on demand and swapped between adjacent nodes, so as to faithfully increase the communication distance. Entanglement swapping is the key building block for the construction of quantum repeaters. The recent experimental breakthrough of quantum memory at telecom wavelength has also been reported ${ }^{45}$. The highly efficient entanglement swapping in this experiment will be an important experimental step toward the realization of quantum repeater protocols.

How to reduce the multi-pair emission. Multi-photon emission is the main reason for the degradation of raw visibilities in our teleportation and entanglement swapping experiments. To obtain high count rates, we need to excite the SPDC with high pump powers, which inevitably lead to stronger multi-photon emission in SPDC. To solve this problem, recently, we propose and demonstrated a new method - increasing the repetition rate of the pump laser using a $10 \mathrm{GHz}$ repetition rate comb laser ${ }^{40}$. With such a high repetition rate, we can maintain the high visibilities at high pump powers. Another important limitation of the SPDC source is its intrinsic photon statistics. This could be efficiently circumvented by using a bright SPDC source with recently proposed heralding protocols such as the one in Ref. 46.

\section{Methods}

Entangled photon source with GVM condition. Our pulsed polarization-entangled photon source is generated from a periodically poled $\mathrm{KTiOPO}_{4}(\mathrm{PPKTP})$ crystal in a Sagnac interferometer configuration. Since the group-velocity-matching (GVM) condition is satisfied ${ }^{47,48}$, the intrinsic spectral purity of the photons is much higher than the conventional schemes. Therefore, there is no need to use narrow bandpass filters to improve the spectral purity ${ }^{49,50}$. The combination of a Sagnac interferometer and the GVM-PPKTP crystal makes our entangled source compact, stable, highly entangled, spectrally pure and ultra-bright ${ }^{19}$. The mean photon numbers per pulse are $\sim 0.1$ in our photon source with a pump power of $\sim 80 \mathrm{~mW}$. The overall detecting efficiency is $\sim 0.2$.

The SNSPDs. Our superconducting nanowire single photon detectors (SNSPDs) have a system detection efficiency (SDE) of around $70 \%$ with a dark count rate (DCR) less than $1 \mathrm{kcps}^{20,21,36}$. The SNSPD also has a wide spectral response range that covers at least from $1470 \mathrm{~nm}$ to $1630 \mathrm{~nm}$ wavelengths ${ }^{36}$. The measured timing jitter and dead time (recovery time) were $68 \mathrm{ps}^{20}$ and $40 \mathrm{~ns}^{51}$.

Theoretical prediction of the entangled sources. The theoretical model of the visibility of the polarization correlation (Fig. 2) including multi-photon emissions is established for example in Refs. 28, 52, 53. For the Sagnac-loop source with mean photon-pair number $2 \mu$ and overall efficiency $\eta$, the visibility is predicted to be

$$
V=\frac{1+\mu}{1+3 \mu+2 \eta(2-\eta) \mu^{2}} .
$$

With $2 \mu=0.1$ and $\eta=0.2$, we have $V=0.912$. Moreover, the model in Ref. 28 can include the imperfection of the PBS (nonzero transmission of the vertically polarized photons). Assuming $1 \%$ of unwanted transmittance of vertical photons at the PBSs, the visibility is degraded to be $V=0.876$. All the experimental raw visibilities in Fig. 2 are around these values, reflecting the validity of our sources.

1. Acín, A. et al. Device-independent security of quantum cryptography against collective attacks. Phys. Rev. Lett. 98, 230501 (2007).

2. Ekert, A. K. Quantum cryptography based on Bell's theorem. Phys. Rev. Lett. 67, 661-663 (1991).

3. Tittel, W., Zbinden, H. \& Gisin, N. Experimental demonstration of quantum secret sharing. Phys. Rev. A 63, 042301 (2001).

4. Gisin, N., Ribordy, G., Tittel, W. \& Zbinden, H. Quantum cryptography. Rev. Mod. Phys. 74, 145-195 (2002). 
5. Briegel, H.-J., Dür, W., Cirac, J. I. \& Zoller, P. Quantum repeaters: The role of imperfect local operations in quantum communication. Phys. Rev. Lett. 81, 5932-5935 (1998).

6. Dür, W., Briegel, H.-J., Cirac, J. I. \& Zoller, P. Quantum repeaters based on entanglement purification. Phys. Rev. A 59, 169-181 (1999).

7. Duan, L.-M., Lukin, M. D., Cirac, J. I. \& Zoller, P. Long-distance quantum communication with atomic ensembles and linear optics. Nature 414, 413-418 (2001).

8. Sangouard, N. et al. Robust and efficient quantum repeaters with atomic ensembles and linear optics. Phys. Rev. A 77, 062301 (2008).

9. Kaltenbaek, R., Prevedel, R., Aspelmeyer, M. \& Zeilinger, A. High-fidelity entanglement swapping with fully independent sources. Phys. Rev. A 79, 040302 (2009).

10. Pan, J.-W., Bouwmeester, D., Weinfurter, H. \& Zeilinger, A. Experimental entanglement swapping: Entangling photons that never interacted. Phys. Rev. Lett. 80, 3891-3894 (1998).

11. Jennewein, T., Weihs, G., Pan, J.-W. \& Zeilinger, A. Experimental nonlocality proof of quantum teleportation and entanglement swapping. Phys. Rev. Lett. 88, 017903 (2001)

12. Marcikic, I., de Riedmatten, H., Tittel, W., Zbinden, H. \& Gisin, N. Long-distance teleportation of qubits at telecommunication wavelengths. Nature 421, 509-513 (2003)

13. de Riedmatten, H. et al. Long-distance entanglement swapping with photons from separated sources. Phys. Rev. A 71, 050302 (2005).

14. Wu, Q.-L., Namekata, N. \& Inoue, S. High-fidelity entanglement swapping at telecommunication wavelengths. J. Phys. B: At. Mol. Opt. Phys. 46, 235503 (2013).

15. Halder, M. et al. Entangling independent photons by time measurement. Nat. Phys. 3, 692-695 (2007).

16. Xue, Y., Yoshizawa, A. \& Tsuchida, H. Hong-Ou-Mandel dip measurements of polarization-entangled photon pairs at $1550 \mathrm{~nm}$. Opt. Express 18, 8182-8186 (2010).

17. Xue, Y., Yoshizawa, A. \& Tsuchida, H. Polarization-based entanglement swapping at the telecommunication wavelength using spontaneous parametric downconversion photon-pair sources. Phys. Rev. A 85, 032337 (2012).

18. Takesue, H. \& Miquel, B. Entanglement swapping using telecom-band photons generated in fibers. Opt. Express 17, 10748-10756 (2009).

19. Jin, R.-B. et al. Pulsed Sagnac polarization-entangled photon source with a PPKTP crystal at telecom wavelength. Opt. Express 22, 11498-11507 (2014).

20. Miki, S., Yamashita, T., Hirotaka, T. \& Zhen, W. High performance fiber-coupled $\mathrm{NbTiN}$ superconducting nanowire single photon detectors with GiffordMcMahon cryocooler. Opt. Express 21, 10208-10214 (2013).

21. Yamashita, T., Miki, S., Terai, H. \& Wang, Z. Low-filling-factor superconducting single photon detector with high system detection efficiency. Opt. Express 21, 27177-27184 (2013).

22. Herbst, T. et al. Teleportation of entanglement over $143 \mathrm{~km}$. arXiv:1403.0009 (2014).

23. Yin, J. et al. Quantum teleportation and entanglement distribution over 100kilometre free-space channels. Nature 488, 185-188 (2012).

24. Yao, X.-C. et al. Observation of eight-photon entanglement. Nat. Photon. 6 , 225-228 (2012).

25. Huang, Y.-F. et al. Experimental generation of an eight-photon GreenbergerHorne-Zeilinger state. Nat. Commun. 2, 546(1-6) (2011).

26. Jin, R.-B., Shimizu, R., Wakui, K., Benichi, H. \& Sasaki, M. Widely tunable single photon source with high purity at telecom wavelength. Opt. Express 21, 10659-10666 (2013).

27. Kim, T., Fiorentino, M. \& Wong, F. N. C. Phase-stable source of polarizationentangled photons using a polarization Sagnac interferometer. Phys. Rev. A 73 012316 (2006)

28. Takeoka, M., Jin, R.-B. \& Sasaki, M. Full analysis of multi-photon pair effects in spontaneous parametric down conversion based photonic quantum information processing. arXiv: 1410.3627 (2014).

29. Hong, C. K., Ou, Z. Y. \& Mandel, L. Measurement of subpicosecond time intervals between two photons by interference. Phys. Rev. Lett. 59, 2044-2046 (1987).

30. Jin, R.-B. et al. Nonclassical interference between independent intrinsically pure single photons at telecommunication wavelength. Phys. Rev. A 87, 063801 (2013).

31. Bouwmeester, D. et al. Experimental quantum teleportation. Nature 390, 575-579 (1997).

32. Pan, J.-W. et al. Multiphoton entanglement and interferometry. Rev. Mod. Phys. 84, 777-838 (2012).

33. Peres, A. Separability criterion for density matrices. Phys. Rev. Lett. 77, 1413-1415 (1996).

34. Aboussouan, P., Alibart, O., Ostrowsky, D. B., Baldi, P. \& Tanzilli, S. Highvisibility two-photon interference at a telecom wavelength using picosecondregime separated sources. Phys. Rev. A 81, 021801 (2010).
35. Bruno, N., Martin, A., Guerreiro, T., Sanguinetti, B. \& Thew, R. T. Pulsed source of spectrally uncorrelated and indistinguishable photons at telecom wavelengths. Opt. Express 22, 17246-17253 (2014).

36. Jin, R.-B. et al. Efficient detection of an ultra-bright single-photon source using superconducting nanowire single-photon detectors. Opt. Commun. 336, 47-54 (2015).

37. Ma, X.-S. et al. Quantum teleportation over 143 kilometres using active feedforward. Nature 489, 269-273 (2012)

38. Kimble, H. J. The quantum internet. Nature 453, 1023-1030 (2008).

39. Khalique, A. \& Sanders, B. C. Long-distance quantum communication through any number of entanglement-swapping operations. Phys. Rev. A 90, 032304 (2014).

40. Jin, R.-B. et al. Efficient generation of twin photons at telecom wavelengths with $2.5 \mathrm{GHz}$ repetition-rate-tunable comb laser. Sci. Rep. 4, 7468 (2014).

41. Gisin, N., Pironio, S. \& Sangouard, N. Proposal for implementing deviceindependent quantum key distribution based on a heralded qubit amplifier. Phys. Rev. Lett. 105, 070501 (2010).

42. Waks, E., Zeevi, A. \& Yamamoto, Y. Security of quantum key distribution with entangled photons against individual attacks. Phys. Rev. A 65, 052310 (2002).

43. Scherer, A., Sanders, B. C. \& Tittel, W. Long-distance practical quantum key distribution by entanglement swapping. Opt. Express 19, 3004-3018 (2011).

44. Cao, Y. et al. Entanglement-based quantum key distribution with biased basis choice via free space. Opt. Express 21, 27260-27268 (2013).

45. Saglamyurek, E. et al. Quantum storage of entangled telecom-wavelength photons in an erbium-doped optical fibre. arXiv: 1409.0831 (2014).

46. Curty, M. \& Moroder, T. Heralded-qubit amplifiers for practical deviceindependent quantum key distribution. Phys. Rev. A 84, 010304 (2011).

47. Grice, W. P. \& Walmsley, I. A. Spectral information and distinguishability in typeII down-conversion with a broadband pump. Phys. Rev. A 56, 1627-1634 (1997)

48. König, F. \& Wong, F. N. C. Extended phase matching of second-harmonic generation in periodically poled $\mathrm{KTiOPO}_{4}$ with zero group-velocity mismatch. Appl. Phys. Lett. 84, 1644 (2004).

49. Eckstein, A., Christ, A., Mosley, P. J. \& Silberhorn, C. Highly efficient single-pass source of pulsed single-mode twin beams of light. Phys. Rev. Lett. 106, 013603 (2011).

50. Gerrits, T. et al. Generation of degenerate, factorizable, pulsed squeezed light at telecom wavelengths. Opt. Express 19, 24434-24447 (2011).

51. Miki, S., Fujiwara, M., Sasaki, M. \& Wang, Z. NbN superconducting single-photon detectors prepared on single-crystal MgO substrates. IEEE Trans. Appl. Superconduct. 17, 285-288 (2007)

52. Sekatski, P. et al. Detector imperfections in photon-pair source characterization. J. Phys. B: At. Mol. Opt. Phys. 45, 124016 (2012).

53. Kuzucu, O. \& Wong, F. N. C. Pulsed Sagnac source of narrow-band polarizationentangled photons. Phys. Rev. A 77, 032314 (2008).

\section{Acknowledgments}

The authors thank K. Wakui, M. Fujiwara, T. Yamashita, S. Miki, H. Terai and Z. Wang for helpful discussion and assistance in experiment. R.-B. Jin thanks X.-H. Bao and H. Jing for valuable suggestions. This work was supported by the Founding Program for World-Leading Innovative R\&D on Science and Technology (FIRST), and Program for Impulsing Paradigm Change through Disruptive Technologies (ImPACT).

\section{Author contributions}

R.J. designed and performed the experiment, collected and analyzed the data, and wrote the paper. M.T. constructed a numerical model and analyzed the data. U.T. performed the experiment. R.S. analyzed the data. M.S. supervised the whole project and wrote the paper All authors contributed to discussion and revision of the manuscript.

\section{Additional information}

Competing financial interests: The authors declare no competing financial interests.

How to cite this article: Jin, R.-B., Takeoka, M., Takagi, U., Shimizu, R. \& Sasaki, M. Highly efficient entanglement swapping and teleportation at telecom wavelength. Sci. Rep. 5, 9333; DOI:10.1038/srep09333 (2015).

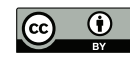

This work is licensed under a Creative Commons Attribution 4.0 International License. The images or other third party material in this article are included in the article's Creative Commons license, unless indicated otherwise in the credit line; if the material is not included under the Creative Commons license, users will need to obtain permission from the license holder in order to reproduce the material. To view a copy of this license, visit http://creativecommons.org/licenses/by/4.0/ 